\title{
Design of Oil Pipeline Monitoring System based on Wireless Sensor Network
}

\author{
Nasheed F. Mossa ${ }^{1}$, Waleed F. Shareef ${ }^{2}$, Faez F. Shareef ${ }^{3}$ \\ ${ }^{1,2}$ University of Technology, Control and System Department, Baghdad, Iraq \\ ${ }^{3}$ University of Technology, Communication Department, Baghdad, Iraq \\ nasheed707@gmail.com,60026@uotechnology.com,30016@uotechnology.com
}

\begin{abstract}
The oil export industry dominates the economy of the world and it depends heavily on oil pipelines. Exposed pipelines are prone to malfunctioning due to intentional or unintentional tampering and vandalism, which is usually caused by damaging form of either knocking or drilling. Continuous structure health monitoring (SHM) of pipelines using conventional methods is difficult and expensive due to the extensive length of the pipelines and the harsh environment. Recent development in printed electronic circuits and microcontrollers open new possibilities in the field of monitoring and have proven their practicality in vibration monitoring process. This paper presents a monitoring system for pipeline heal of the structure based on the wireless sensor network. The system senses the pipeline vibration and relays the data to a base station for the procession. A WSN consists of three nodes is designed and implemented. Each node is built around 32-bit ARM core microcontroller, and equipped with an accelerometer to measure the pipeline vibration. The measurements of each sensor are collected wirelessly through ZigBee protocol to a base station. Results on a $2 \mathrm{~m}$ pipeline sample show the ability of the system to precisely detect damaging events e.g. knocking and drilling to the pipeline.
\end{abstract}

Index Terms - WSN, structure health monitoring, MEMS, oil pipeline.

\section{INTRODUCTIONS}

The recent advances in the field of communication transceivers, microcontrollers, and MicroElectro-Mechanical Systems (MEMS) helped the emergence of a new type of wireless network for sensing and monitoring system called wireless sensors networks. These networks operate in an ad hoc manner, i.e. they do not require a preceding setup or a supporting infrastructure. They utilize new communications protocols built for small range network to cooperatively monitor and record measurements from phenomena of interest, then relay the recorded measurements to a base station. By using the wireless channel to communicate between the phenomena location and the base station, wireless sensor networks offer a reliable and simple scheme for deployment, control, and maintenance [1].

Wireless Sensor Network (WSN) arrangements are in effect dynamically utilized in observing applications, for example, [2] who suggested a WSN for monitoring the environments including recorded data for any parameters such as lighting, gas, motion, and distance. a facility of interfacing of MATLAB with the Arduino microprocessor has exploiting to implement a flexible network. The data of each sensor is collected wirelessly exploiting the ZigBee protocol. In [3] a design for a wireless node with a vibration sensor is proposed for online monitoring of industrial machinery. The main focus is to design a wireless condition monitoring system to detect and diagnose the fault in mechanical transmissions systems (gears and bearings) using vibration signal analysis. The sensor node was used 
to detect faults in an industrial robot joint. Communication through wireless ZigBee protocol was added to the used microcontroller (DUE board) by using both Arduino wireless shield and an XBee wireless module. WSNs has been applied to use for underground pipeline monitoring [4]. Here a pressure/acoustic and soil properties sensors used for detection and localize leakage in an underground pipeline, The measurements were taken by the underground sensors out of pipelines are transmitted by utilizing the magnetic induction (MI)- upon on wireless underground sensors networks. In [5] a measurement system using RFID Tag integrated with an accelerometer for infrastructure health state monitoring was presented. The researchers utilize semi-passive radio-frequency identification (RFID) tag to design a measurements system for remotely monitoring dynamic acceleration of infrastructure. In [6], a monitoring system was applied to an assessment of the dynamic behavior of railway catenary systems. The researchers explore the development of a wireless sensor system for examining the structural behavior of existing railway catenary sections while the pipeline is in operation. The sensors measure acceleration and rotational velocity in and around three axes.

This paper presents the design and implementation of a minimal cost wireless sensor node for structural health state monitoring of oil pipeline. The monitoring system based on measuring the vibration of the pipe and then Data relay and data collection performed to send the measurements wirelessly to a centralized PC station using the ZigBee protocols based upon IEEE 802.15.4 report as an efficient communication link to detect/monitor the pipeline health state. Section 2 presents the background for oil pipeline specifications, while Section 3 presents the wireless node design in term of a hardware component and wireless protocol. Section 4 shows the details of the system setup. The results discussion are given in Section 5, and Section 6 concludes the paper.

\section{OIL PIPELINE SPECIFICATION AND NATURAL FREQUENCY}

The pipes used for the transportation of petroleum products are made of carbon steel, which is highly resistant to corrosion and rust. These pipelines are used as external transport lines between cities or as distribution lines within the main stations in each city. Pipeline diameters range from $25 \mathrm{~mm}$ within the internal transport to $350 \mathrm{~mm}$ in the external transport. In addition, the thickness of the pipe wall is proportional to its diameter [7]. The specification of the pipe used in this work shown in Table I below:

Table 1. Oil Pipeline Specification [7]

\begin{tabular}{cccccc}
\hline Pipe martial type & Length & Outer diameter & Inner diameter & Wall thickness & Weight \\
\hline Carbon steel & $2.0 \mathrm{~m}$ & $114 \mathrm{~mm}$ & $101.4 \mathrm{~mm}$ & $6.3 \mathrm{~mm}$ & $16.07 \mathrm{~kg} / \mathrm{m}$ \\
\hline
\end{tabular}

Obtaining the normal mode information for a structure is a first step in assessing the dynamic behavior of a structure under the applied external body, surface, or boundary forces. The normal modes of vibration for the structure can be used to estimate structure response when the structure is loaded by force fields [8].

The number of natural frequencies of vibration of any system equals the number of degrees-offreedom. So, any system having distributed parameters have infinite numbers of natural frequencies. At a given time, such a system commonly vibrates with an appreciable amplitude at only one frequency or limited numbers of frequencies [9]. The solution to the problem of free vibration in any system would require the determination of all the natural frequencies and of the mode shape associated with each. "In practice, it often is necessary to know only a few of the natural frequencies. Usually, the lowest frequencies are the important" [9]. Therefore, the natural frequency of the pipeline used in this work can be calculated before any external force is applied to it by the following formula [10]: 


$$
F_{n}=\frac{1}{2 \pi} \cdot 22 \cdot 4 \cdot \sqrt{\frac{E I}{\mu L^{4}}}
$$

where:

$\mathrm{Fn}=$ pipe natural frequency $(\mathrm{Hz})$

$\mathrm{E}=$ Young's modulus of elasticity (200GPa or 30E6psi for steels - approximately but close enough)

$\mathrm{I}=4$ th polar moment of inertia of pipe $\left(0.049^{*}\left[\mathrm{OD}^{\wedge} 4-\mathrm{ID}^{\wedge} 4\right]\right)$ in inches or in meters

$\mu=$ pipe mass per unit length $\mathrm{lbs} / \mathrm{inch}$ or $\mathrm{kg} / \mathrm{m}$ here equal to $1.8 \mathrm{~m}$.

$\mathrm{L}=$ the distances between pipe supports (inches or meters)

By using the information in Table 1 and according to the formula above, the natural frequency of the pipe used in this work was calculated and it is equal to $130 \mathrm{~Hz}$.

In order to choose the suitable hardware components (Sensors, Microprocessors, and Radio Frequency communication units) for this work the sampling frequency must obtain first. According to the Shannon theorem [11], the minimum sampling rate equals two samples per period of the maximum frequency component for the signal. Therefore the minimum sampling rate to capture the natural frequency of the selected pipe is $260 \mathrm{~Hz}$. In this paper, the sampling frequency is set to $400 \mathrm{~Hz}$ as explained in the next sections.

\section{SENSOR NODE DESIGN}

The sensor node consists of four main units as illustrated in Figure 1. These units are processing (microcontroller), communications, sensing, and power unit. The sensing unit commonly consists of one or more sensors and analog to digital converters (ADCs). The processing units typically are based around a microcontroller or microprocessor with data storage unit (memory), to collect and process the data locally. The sensors detect the phenomenon parameter of interest and generate analog signals based on this parameter. These signal are then fed to an ADC's that convert the analog signals to digital signal to be captured by the processing unit. The communication unit is an RF transceiver for performing data transmissions and reception over a radio channel. The power unit is typically made of batteries, yet many applications depend on another source such as a solar panel. However, batteries usually offer low cost, small size, and high capacity source of power.

The next subsections discuss the specifications of the selected microcontroller, transceiver accelerometer, and the power source of the node and the reasoning behind this selection.

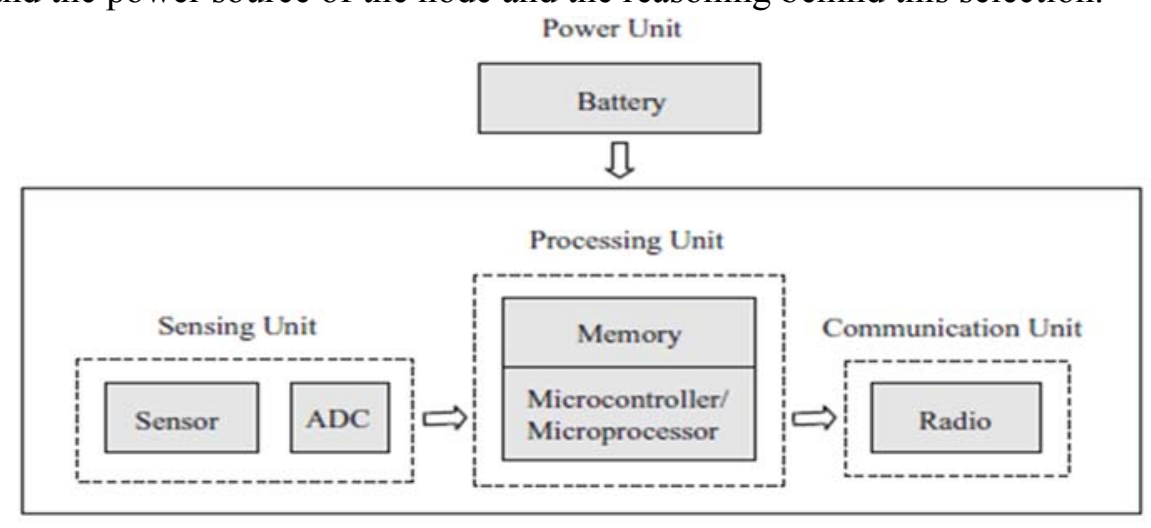

Fig.1. ARCHITECTURE OF WIRELESS SENSOR NODE HARDWARE [1]

\section{A. SELECTION OF SENSOR UNIT: ACCELEROMETER}

Accelerometers are widely utilized for fault detection in many machines due to their reliable operation, precision, and sensitivity. They are electromechanical devices that convert the mechanical signals, such as vibrations and forces, to electrical signals, and. MEMS (Micro-electro-mechanical- 
sensors) accelerometers consume less power, have the small form factor, and weigh less, than another vibration sensor. All these features in addition to low cost make the MEMS accelerometer more suitable for wireless systems applications. In this paper, a three axes accelerometer is used to monitor the pipeline vibrations. Among several available commercial accelerometers, the 3-axis ADXL345 MEMS accelerometer has been chosen, since it is widely used in the field of vibration and motion research field. Also, its operating conditions and specifications are well documented.

The ADXL345 as shown in Figure 2, is [12] a small, thin, developed by Analog Devices. Ultralow power: as low as $23 \mu \mathrm{A}$ in measurements mode and $0.1 \mu \mathrm{A}$ in standby mode. The ADXL345 operates on a supply voltage (2.0 - 3.6 volt), 3-axis accelerometer with resolution (10 bit) measurement at ranges of $\pm 2, \pm 4$, and $\pm 8 \mathrm{G}$, ( $\mathrm{G}$ is the acceleration due to earth gravity which approximately equals to 9.807 $\mathrm{m} / \mathrm{s}^{2}$ ), and with resolution of 13-bit measurements at range up to $\pm 16 \mathrm{G}$. The sensor digital output is packed in two bytes in the 2's complement format. The board uses either an SPI or I2C as a digital interface. The output data rate between 0.1 and $3200 \mathrm{~Hz}$. This is very suitable to the sampling rate needed for this work.

\section{B. SELECTION OF MICROCONTROLLER}

The processor unit represents is what gives intelligence to the WSN node. The selection of a processor unit for WSNs are highly dependent on the application's class [3]. The key requirement that should be considered when selecting a processor unit for building a WSN node is to achieve a balance between the cost and processing speed. Other requirements involve physical size, required memory size, energy consumption, and support for peripherals. Currently, many companies provide an off-shelf microcontroller/microprocessor board for research and amateur users, for instance, Arduino, Microchip, and Texas Instruments. Arduino board was observed to be appropriate for this work, as it meets the design requirements explained above. This board is also compatible with a wide range of electronic components and has a large community supports which make the development process easier.

"Arduino is an open-source electronics platform based on easy-to-use hardware and software" [13]. Arduino DUE shown in figure 3, based upon 32-bits, Atmel SAM3X8E ARM Cortex-M3 processor has features both of microprocessor (in term of speed and computational power) and microcontroller (in term of power consumption and board size). Also, based on the pipeline and the sensor specifications listed in table 1 and in [12], we found that the DUE board is sufficient for pipeline monitoring job.

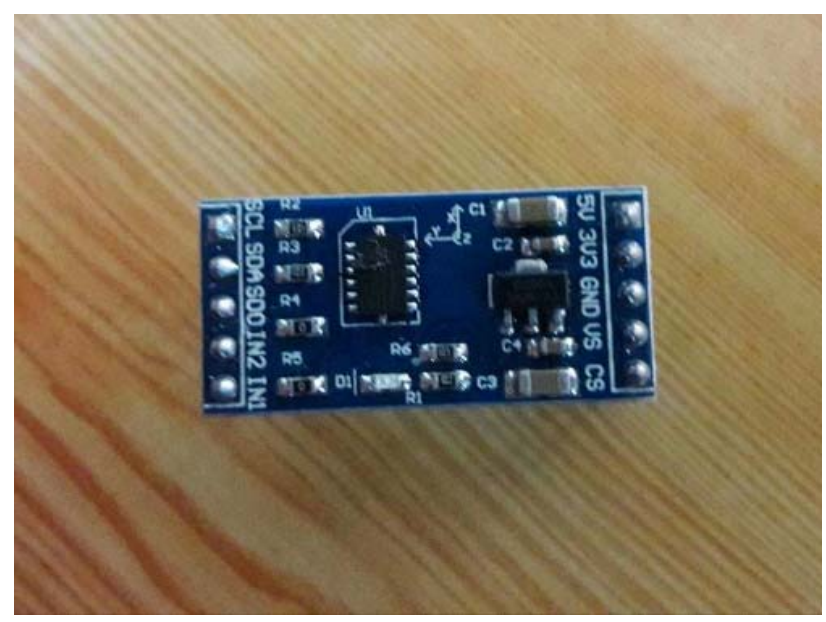

FIG. 2. ADXL345 ACCELEROMETER 


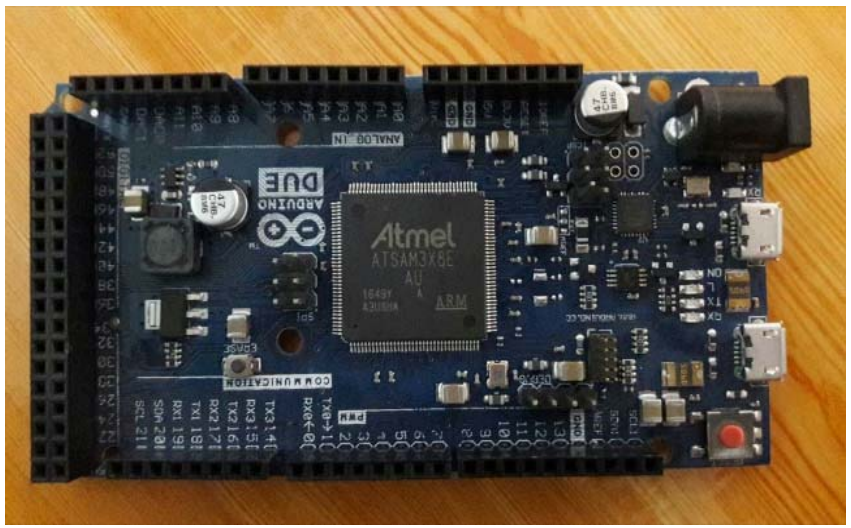

FIG. 3. ARDUINO DUE MICROCONTROLLER BOARD

The main characteristics of Arduino DUE are listed in Table 2. More details are available in [14].

TABLE 2. ARDUINO DUE CHARACTERISTICS [14]

\begin{tabular}{|c|c|}
\hline Microcontroller type & AT91 SAM3X8E \\
Operating Voltage & $3.3 \mathrm{~V}$ \\
DC Current of 3.3V Pin & $800 \mathrm{~mA}$ \\
Size of Flash Memory & $512 \mathrm{~KB}$ available to user for applications \\
Size of SRAM & $96 \mathrm{~KB}$ (two banks: $64 \mathrm{~KB}$ and 32 KB) \\
Clock Speeds & $84 \mathrm{MHz}$ \\
\hline
\end{tabular}

\section{SELECTION OF WIRELESS TECHNOLOGY}

Recent research in the field of wireless sensor networks relays heavily on 802.11 and 802.15.4 protocols or their commercial counterparts, ZigBee and Wi-Fi protocols to implement the communication channel [3]. General ZigBee consumes less power and provides less communication range than Wi-Fi, hence it will be used for this project. ZigBee is a standard designed by the ZigBee Alliance, which is a group of companies working together to low-power, empower reliable, and costeffective wireless networks [15]. Additionally, ZigBee is designed to provide High security, Simple protocol, and global implementation. Operate on IEEE 802.15.4 physical radio specifications and operate in unlicensed bands including $2.4 \mathrm{GHz}$. Transmission distances range from 10 to 100 meters. ZigBee supports more than one network topology such as point-to-point, point-to-multipoint, and mesh networks and lets up to 65,000 nodes per network and provides wireless communications speeds up to 250 kbps. The XBee module (XBee Digi Internationals) will be used as an RF transceiver, as it meets the necessities of this work. It is also compatible with Arduino DUO board. XBee is a wireless module designed for applications that require relatively low data rate and long-range communication while maintaining low power consumptions. Three nodes were used in this work, two nodes were programmed as a sensor node and one programmed as a coordinator which is an XBee radio module connected to the computer via USB cable. The power unit used in this work is a lithium 9-volt battery.

\section{SYSTEM SETUP}

The proposed oil pipeline monitoring system based on WSN is shown in Figure 4. The system consists of two sensor nodes mounted at the end of the $2 \mathrm{~m}$ carbon steel oil pipeline, and one coordinator connected to the computer. The distance between sensors is $1.7 \mathrm{~m}$. based on the specifications discussed in the previous sections, each node should be of small size and easily fastened. The node should also be 
of a lightweight to avoid adding extra loadings on the pipe. The pipe mounted on one stand in each end. In this work, real-time vibration signals resulting from the damaging activity on the pipe are captured by the accelerometer of each node and transmitted wirelessly to the coordinator node. The position of (X-Y-Z) axis of the installed accelerometer for each node on the pipe shown in figure 5. There are many damaging activities that may affect the exposed (above earth) oil pipeline structure, for example, ramming, picking, drilling, pipe knocking, forklift working.

The sampling rate for the accelerometer is set to $400 \mathrm{~Hz}$ output data rate (200 Hz bandwidth). That is appropriate to capture the natural frequency of the selected pipe. The resolution of each data sample is set to (10 bit) measurement within a total ranges of \pm 2 normal power mode. The I2C digital interface protocol was used to connect the accelerometer with the Arduino board. The testing for the system was performed by capturing the vibrating real-time signals in a three-axis of the accelerometer, as a result of knocking the pipe by hand hammer and drilling the pipe.

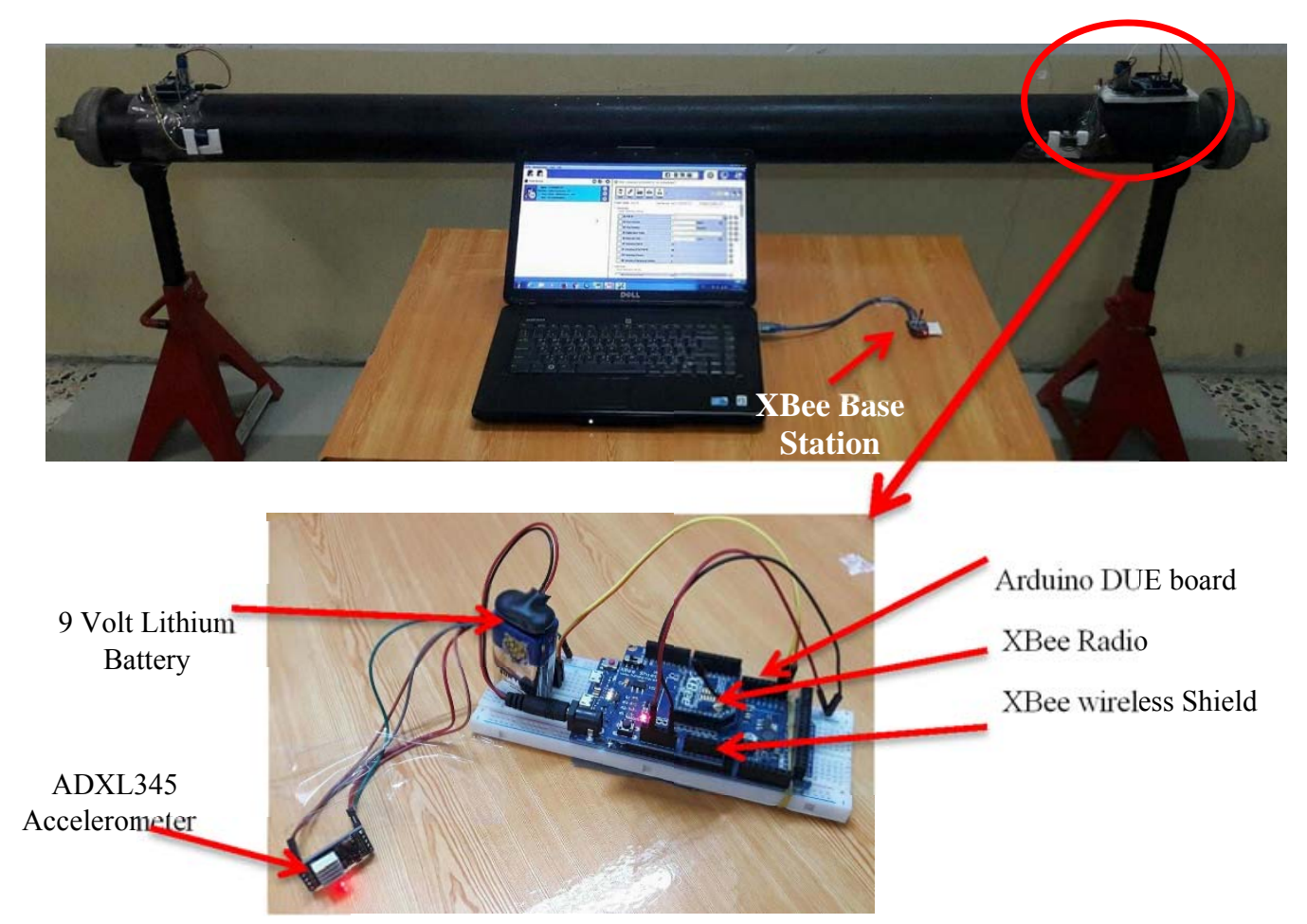

FIG. 4. WIRELESS SENSOR NODE HARDWARE EXPERIMENTAL SETUP

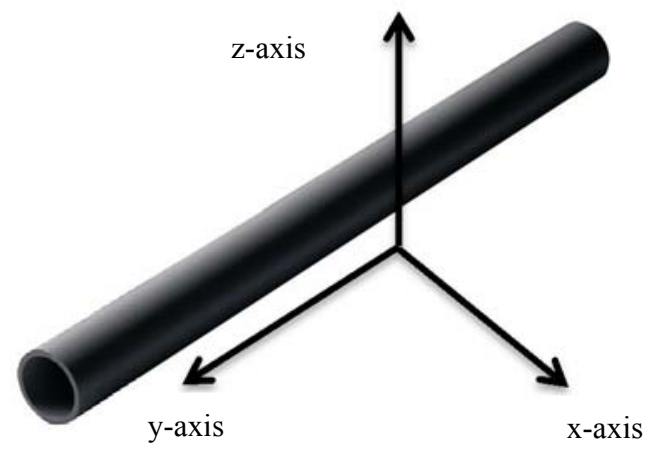

Fig. 5. ACCELEROMETER LAYOUt RELATIVE TO THE PIPE 


\section{RESULTS AND DISCUSSIONS}

For exposed pipeline (above the earth) intentional or unintentional tampering can take several damaging forms. It is worth to mention that the system is proposed for the detection of the tampering event effects on the pipe while it happens, by comparing it to the previously recorded normal operation state. For the purpose of this paper, we subjected the pipe to both knocking and drilling events. These events were applied using a hammer and a handheld electric drill. The result recorded in this section for each sensor individually. Figure 6 below shows the reading of each sensor that displayed on the PC station. Each sensor is indicated by three values for three axes. The value of the first sensor named as $(\mathrm{X} 1, \mathrm{Y} 1, \mathrm{Z} 1)$ and the values of the other sensor is $(\mathrm{X} 2, \mathrm{Y} 2, \mathrm{Z} 2)$.

Since the readings were close to each sensor node was approximated to each other, we relied on the average measurements of nodes. Figure 7 shows that the process of knocking the pipe three times in average time equal to approximately (11 seconds). The output real-time signals from the accelerometer in gravity units. When no event on the pipe, the average normal values of $\mathrm{X}, \mathrm{Y}, \mathrm{Z}$-axis amplitude is $0.095 \mathrm{G}, 0.04 \mathrm{G}$, and $1.07 \mathrm{G}$ respectively.

The values for the $\mathrm{Z}$ axes was shifted $1 \mathrm{G}$ due to ear th gravity. When knock event is applied these values increase to $1.3 \mathrm{G}, 0.25 \mathrm{G}$ and $1.75 \mathrm{G}$. From this results of measurements the maximum value of acceleration result from knocking occurred in $\mathrm{Z}$-axis because of the event of knocking Perpendicular to this axis. The average time is taken to vibrate the pipe by each one knock approximately (1 second).

Figure 8 below shows that the process of drilling the pipe two times in total average time equal to (50 seconds). When no event on the pipe, the average normal values of $\mathrm{X}, \mathrm{Y}, \mathrm{Z}$-axis amplitude is $0.045 \mathrm{G}, 0.04 \mathrm{G}$, and $1.07 \mathrm{G}$ respectively. The values for the $\mathrm{Z}$ axes was shifted $1 \mathrm{G}$ due to earth gravity. During the pipe drilling, the accelerometer measurement values were between $\pm 1 \mathrm{G}$ for $\mathrm{X}$-axis and $\mathrm{Y}$ axis and $2 \mathrm{G}$ for $\mathrm{Z}$-axis. The average time is taken to vibrate the pipe by each one drilling process approximately (15 seconds).
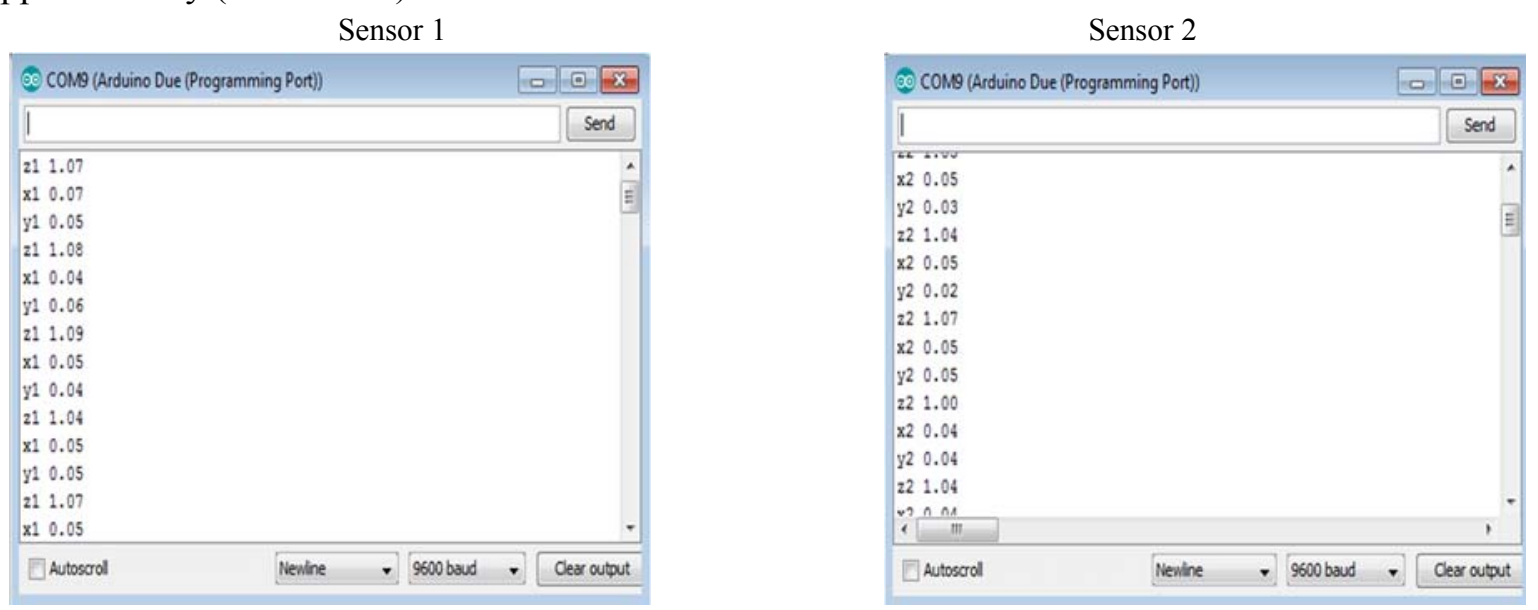

FIG. 6. THE READING OF SENSORS IN SERIAL MONITOR OF ARDUINO 

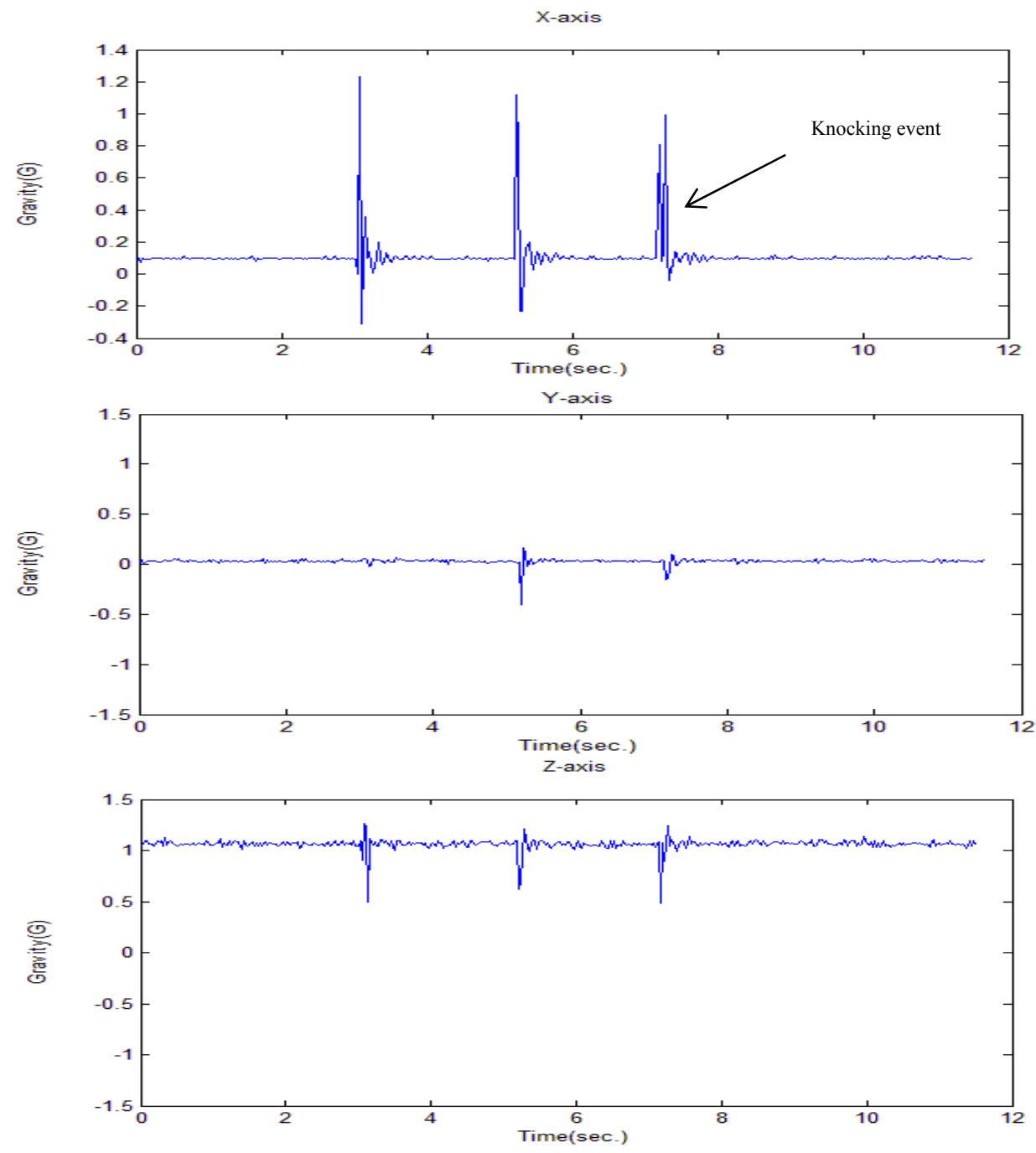

Fig. 7. MEASUREMENTS OF ACCELEROMETER WHEN KNOCKING THE PIPE 

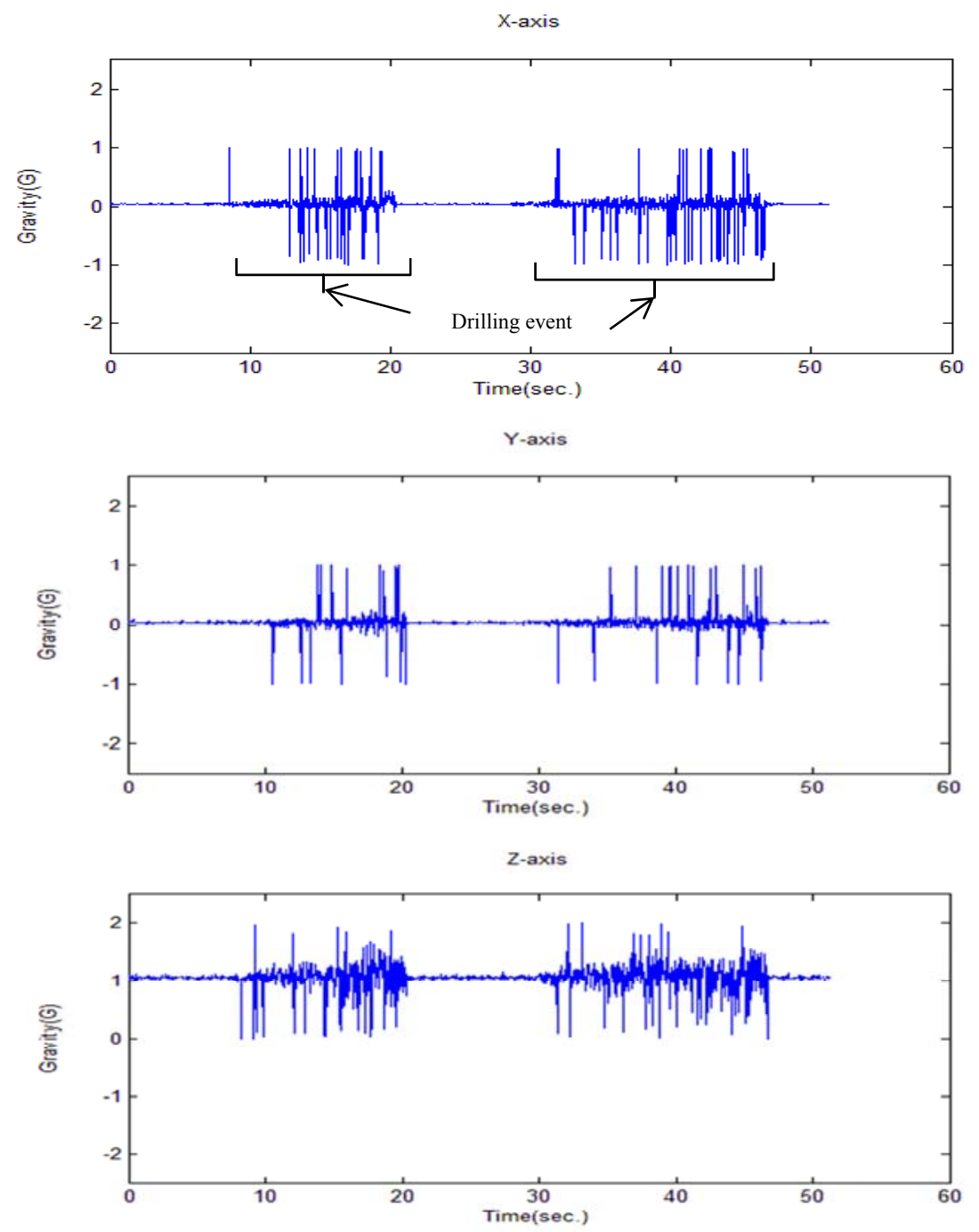

Fig. 8. MEASUREMENTS OF ACCELEROMETER WHEN DRILLING THE PIPE

\section{CONCLUSION}

This paper presents a system to measure and wirelessly collect vibration measurements from a pipeline to detect any ongoing damaging events. The proposed system successfully maintains a tradeoff between limited resources of low-cost sensor nodes and data quality as required for the detection. A network of three nodes (two sensor nodes and one acts as a base station) is designed and implemented. Each sensor nodes is consist of a 32-bit ARM core microcontroller, 3-axis ADXL accelerometer, and ZigBee RF transceiver. The sensor nodes were mounted on carbon steel oil pipe with a length of $2.0 \mathrm{~m}$. Each node transfers the accelerometer measurements wirelessly to the base node that is connected to the computer for data collection and display. The Arduino DUE board was selected as the main part of the wireless sensor unit because it has the features of both microprocessors (in term of speed and computational power) and microcontroller (in term of power consumption and board size). During the experimental test, damaging events of knocking (with a hammer) and drilling (with an electrical handheld drill) was applied to the pipeline sample. The signals captured by using the accelerometer showed sufficient sensitivity to distinguish the damaging events. Results show that using the Arduino DUE boards offers functional accuracy that is suitable for detecting damaging effects. While using the ZigBee wireless protocol guarantee less power consumption and reliable RF communication. 


\section{REFERENCES}

[1] Rastko R. Selmic Vir V. Phoha and Abdl Srwadda,"Wireless Sensors Networks Security, Coverage, and Localization", Springer Internationals Publishing AG 2016.

[2] Dr. Mahmood F. Mosleh, Dr. Rashid A. Fayadh, and Shahad A. Hamid," Design and Implementation of Wireless Sensors Network Based on MATLAB Interface with Arduino", International Journal of Engineering and Technology (IJET), Vol 9 No 4 Aug-Sep 2017.

[3] Ala Abdulhady Jabr, Robert Bicker," Design of Wireless Sensors Node for Vibration Monitoring of Industrial Machinery", Internationals Journal of Electrical and Computers Engineering (IJECE), Vol. 6, No. 2, April 2016, pp. 639 653.

[4] Zhi Sun, Pu Wanga, Mehmet C. Vuran, Mznah A. Al-Rodhaan, Abdullah M. Al-Dhelaanb, and Ian F. Akyildiz, " MISEPIPE: Magnetic induction based wireless sensors networks for underground pipelines monitoring", Ad Hoc Networks 9 (2011) 218-227 Elsevier B.V.

[5] Devaka Jayawardana and Ranjith Liyanapathirana,"Measurements System With Accelerometer Integrated RFID Tag for Infrastructure Health Monitoring", IEEE Transactions on instrumentation and measurement 0018-9456, 2015 IEEE.

[6] Petter Nåvika, Anders Rønnquista and Sebastian Stichel," A wireless railway catenary structural monitoring systems: Full scale case study", Case Studies in Structural Engineering 6 (2016) 22-30 Published by Elsevier Ltd.

[7] Iraqi Ministry of Oil, Oil Pipeline Company, document of specifications of the oil pipelines.

[8] Allan G. Piersol, Thomas L. Paez, "Harris' shock and vibration Handbook", sixth edition, 2010.

[9] Cyril M. Harris, Allan G. Piersol ," Harris' shock and vibration Handbook”, fifth edition,2002.

[10] Piping vibration - calculating natural frequency of a pipe, https://www.petroskills.com/blog/entry/piping-vibrationcalculate-natural-frequency.

[11] Robert J. Marks II," Introduction to Shannon Sampling and Interpolations Theory", Springer-Verlag, New York, 1991.

[12]3-axis accelerometers ADXL345 Datasheet Available in https://www.sparkfun.com/datasheets/Sensors/Accelerometers/ADXL345.pdf.

[13]General information about Arduino Microcontroller Available in the site http://www.arduino.ccenGuideIntroduction.

[14] Datasheet of Arduino DUE board Available in site https://store.arduino.cc/usa/arduino-due 2017.

[15] XBee Zigbee Mesh Kit, Radio Frequency (RF) Modules. Available in site http://www.digi.comresourcesdocumentationdigidocspdfs90001942-13.pdf. 